\title{
Gravitational Waves and Degrees of Freedom in Higher Derivative Gravity
}

\author{
Patric Hölscher* \\ Fakultät für Physik, Bielefeld University, Postfach 100131, 33501 Bielefeld, Germany
}

\begin{abstract}
We study the degrees of freedom of the metric in a general class of higher derivative gravity models, which are interesting in the context of quantum gravity as they are (super)renormalizable. First, we linearize the theory for a flat background metric in Teyssandier gauge for an arbitrary number of spacetime dimensions $D$. The higher-order derivative field equations for the metric perturbation can be decomposed into tensorial and scalar field equations resembling massless and massive wave equations. For the massive tensor field in $D$-dimensions we demonstrate that the harmonic gauge condition is induced dynamically and only the transverse modes are excited in the presence of a matter source. For the special case of quadratic gravity in four-dimensional spacetime, we show that only the quadrupole moment contributes to the gravitational radiation from an idealized binary system.

PACS numbers: 04.30.-w, 04.50.Kd
\end{abstract}

\section{INTRODUCTION}

General relativity (GR) as the standard theory of gravity works very well on solar system distance scales [1]. Nevertheless, considering only the luminous matter, GR cannot explain several astrophysical [2] as well as cosmological [3] phenomena. This led to the introduction of dark matter as a new particle, which interacts only very weakly with other standard model particles, but couples to gravity. Besides that, the small value of the cosmological constant (compared to the zero-point energy density from particle physics) is not understood [4-6].

Further, it seems to be impossible to combine GR with quantum mechanics in the ultraviolet regime. A perturbative quantization of GR leads to divergences, which cannot be renormalized $[7,8]$. This means a quantum theory of GR is nonpredictive. In the course of solving the renormalization problem, a class of fourthorder gravity theories [9], which improve on the renormalization behavior [10], were investigated. Later is was shown that theories with six or more derivatives are even superrenormalizable [11-13]. Unfortunately, the (super)renormalization property comes along with the problem of Ostrogradsky instabilities [14, 15] manifesting as ghost fields, which are states with negative kinetic energy. The appearance of ghost states is a general feature of theories with higher derivatives and could be used as an argument to invalidate these theories. Nevertheless, as the (super)renormalizability seemed so promising, people were strongly motivated to resolve the ghost problem, e.g., by modifying the quantization scheme [1625] or by nonlocal theories [26-29]. But still there is no unanimous opinion about the ghost issue [30]. Hence, we do not treat the ghost problem in this work.

However, even if it turns out that the ghost problem cannot be solved, it is interesting to consider theories with higher derivatives as effective theories in the low-

\footnotetext{
* patric.hoelscher@physik.uni-bielefeld.de
}

energy regime of more fundamental theories, like string theory, which do not suffer from ghost instabilities and renormalization problems [31-33].

Many tests have been performed to constrain theories of modified gravity. Besides the direct detection of gravitational waves by the aLIGO/VIRGO interferometers [34-39], one can use the indirect detection of gravitational waves by measuring the decrease of the orbital period of stellar binary systems. This decrease of the orbital period agrees to high precision with the prediction of GR [40], and hence it is ideal to test theories of modified gravity. Besides that, the first detection of gravitational waves (GWs) from a binary neutron star merger GW170817 with electromagnetic follow-up signals coming from GRB 170817A [39, 41, 42] in a large range of the energy spectrum put strong constraints on the speed of the gravitational waves and hence on many theories of modified gravity [43-50].

In this work, we want to analyze aspects of the gravitational wave solutions in generalized higher-derivative gravity [11] (see also [25, 51-53] for studies of measurable effects in the low-energy regime). We structure this work in the following way. In Sec. II, we introduce the theory of higher-derivative gravity and derive the linearized field equations for the gravitational field containing a massive spin-0, a massless spin-2, and a massive spin-2 field (the massless spin-2 obeys the same field equation as in GR). After that, in Sec. III using the method of Green's function, we analyze the massive spin- 0 and the massive spin-2 fields in the special case of quadratic gravity. To see how these modes affect the gravitational radiation, we study the solutions in the presence of a binary system in circular motion and in the Newtonian limit. For the massive spin-2 field we also analyze the number of degrees of freedom (d.o.f.) that are excited by a matter source. Finally, we summarize and conclude.

Throughout the paper, we use $c=\hbar=1$. Latin indices run from 1 to $D$ and greek indices run from 0 to $D$, where $D$ is the number of spacetime dimensions. Repeated indices are implicitly summed over. $\mathbf{x}$ denotes the $(D-1)$-dimensional spatial vector. Further conventions 
are defined in Appendix A.

\section{FOURTH-ORDER DERIVATIVE GRAVITY}

In this work we study a general class of higherderivative theories of gravity, which are invariant under general coordinate transformations and include terms up to quadratic order in curvature tensors. In such a case the most general $D$-dimensional $(D \geq 3)$ action is given by $[11]$

$$
\begin{aligned}
S=\int d^{D} x \frac{\sqrt{-g}}{64 \pi G}[ & -4 \epsilon R+R F_{1}(\square) R+R_{\mu \nu} F_{2}(\square) R^{\mu \nu} \\
& \left.+R_{\mu \nu \rho \sigma} F_{3}(\square) R^{\mu \nu \rho \sigma}\right]+S_{m},
\end{aligned}
$$

where $G$ is Newton's constant, $g=\operatorname{det}\left(g_{\mu \nu}\right)$ is the determinant of the metric and $S_{m}$ is the matter action. $R_{\nu \rho \sigma}^{\mu}, R_{\mu \nu}$ and $R$ are the Riemann tensor, Ricci tensor and Ricci scalar defined in Appendix A. $\epsilon$ is a parameter that takes the values $\pm 1 . F_{1}(\square), F_{2}(\square)$ and $F_{3}(\square)$ are polynomial functions of the covariant d'Alembert operator $\square=g^{\mu \nu} \nabla_{\mu} \nabla_{\nu}$ defined by $F_{i}(\square)=\sum_{k=0}^{n} \alpha_{i_{k}} \square^{k}$, where $n$ is an arbitrary but finite nonnegative integer and $\alpha_{i_{k}}$ are real coefficients with a canonical mass dimension $M^{-2(k+1)}$. $\square^{k}=\left(\nabla_{\rho} \nabla^{\rho}\right)^{k}$ acts on tensors in the usual sense. If $F_{1}, F_{2}$, and $F_{3}$ are independent of the d'Alembert operator (corresponding to $n=0$ ),

(1) reduces to the action of quadratic gravity (see, e.g., $[54,55])$. As a special case of quadratic gravity, for $D=4, \epsilon=-1, F_{1}=128 \pi G \alpha_{g} / 3, F_{2}=-128 \pi G \alpha_{g}$, and $F_{3}=0$ the action reduces to conformal gravity [56]. Note also that for $D=4, \epsilon=+1$, and $F_{1}=F_{2}=F_{3}=0$ one recovers the Einstein-Hilbert action.

\section{A. Linearized Wave Equations}

We are interested in the linearized version of this theory in a flat Minkowski background spacetime and hence we write

$$
g_{\mu \nu}=\eta_{\mu \nu}+h_{\mu \nu},
$$

where $h_{\mu \nu}$ represents a small metric perturbation and the d'Alembert operator reduces to $\square=\eta^{\mu \nu} \partial_{\mu} \partial_{\nu}$.

In linearized theory there is a useful relation [58]

$$
\begin{aligned}
R_{\mu \nu \rho \sigma} F_{3}(\square) R^{\mu \nu \rho \sigma}= & 4 R_{\mu \nu} F_{3}(\square) R^{\mu \nu}-R F_{3}(\square) R+\partial \Omega \\
& +\mathcal{O}\left(h^{3}\right),
\end{aligned}
$$

where $\partial \Omega$ denotes a surface term, which does not contribute to the field equations for appropriate boundary conditions. We also neglect terms cubic in the metric perturbation, since these terms do not contribute to the linearized field equations. This shows that by a redefinition of $F_{1}(\square)$ and $F_{2}(\square)$ we can set $F_{3}(\square)=0$. For this reason we drop the $F_{3}$-term in the further analysis.
Expanding (1) to second order in $h_{\mu \nu}\left(\right.$ for $\left.F_{3}=0\right)$ and varying with respect $h_{\mu \nu}$, we find the linearized field equations

$$
\begin{aligned}
& \left(\epsilon+\frac{1}{4} F_{2}(\square) \square\right) G_{\mu \nu}^{(1)}+\left(\frac{1}{2} F_{1}(\square)+\frac{1}{4} F_{2}(\square)\right) \\
& \times\left(\eta_{\mu \nu} \square-\partial_{\mu} \partial_{\nu}\right) R^{(1)}=-8 \pi G T_{\mu \nu},
\end{aligned}
$$

where $G_{\mu \nu}=R_{\mu \nu}-1 / 2 \eta_{\mu \nu} R$ is the Einstein tensor and ... (1) denotes quantities that are linear in $h_{\mu \nu}$. We observe that the field equations do not contain derivatives of the $F_{i}$ 's. This is a result of the linearization because covariant derivatives reduce to partial derivatives which commute with the $F_{i}$ 's. This is not true for the nonlinear field equations (for details, see [59]). For a list of the linearized curvature tensors, see Appendix A. The matter energy-momentum tensor is defined by

$$
T_{\mu \nu}=-\frac{2}{\sqrt{-g}} \frac{\delta S_{m}}{\delta g^{\mu \nu}} .
$$

The trace of (4) is given by

$$
\begin{aligned}
& \left(\frac{1}{2} F_{1}(\square)+\frac{1}{4} F_{2}(\square)\right) \square R^{(1)} \\
& \quad=-\frac{8 \pi G}{(D-1)} T+\frac{D-2}{2(D-1)}\left(\epsilon+\frac{1}{4} F_{2}(\square) \square\right) R^{(1)},
\end{aligned}
$$

where $T=\eta^{\mu \nu} T_{\mu \nu}$ is the trace of the matter energymomentum tensor. Using (6) one can rewrite (4) as

$$
\begin{aligned}
& \left(\epsilon+\frac{1}{4} F_{2}(\square) \square\right)\left(R_{\mu \nu}^{(1)}-\frac{1}{2(D-1)} \eta_{\mu \nu} R^{(1)}\right) \\
& -\left(\frac{1}{2} F_{1}(\square)+\frac{1}{4} F_{2}(\square)\right) \partial_{\mu} \partial_{\nu} R^{(1)} \\
& =-8 \pi G\left(T_{\mu \nu}-\frac{1}{D-1} \eta_{\mu \nu} T\right) .
\end{aligned}
$$

Now, it is convenient to define

$$
\begin{aligned}
Z_{\mu} \equiv & -\left(\epsilon+\frac{1}{4} F_{2}(\square) \square\right) \partial^{\alpha} \bar{h}_{\alpha \mu} \\
& -\left(\frac{1}{2} F_{1}(\square)+\frac{1}{4} F_{2}(\square)\right) \partial_{\mu} R^{(1)}
\end{aligned}
$$

where $\bar{h}_{\mu \nu}=h_{\mu \nu}-1 / 2 \eta_{\mu \nu} h$ is the trace-reversed metric perturbation and $h=\eta^{\mu \nu} h_{\mu \nu}$. Using (8) we can bring (7) to the form

$$
\begin{aligned}
& \left(\epsilon+\frac{1}{4} F_{2}(\square) \square\right)\left(\frac{1}{2} \square h_{\mu \nu}-\frac{1}{2(D-1)} \eta_{\mu \nu} R^{(1)}\right) \\
& +\frac{1}{2}\left(\partial_{\nu} Z_{\mu}+\partial_{\mu} Z_{\nu}\right)=-8 \pi G\left(T_{\mu \nu}-\frac{1}{D-1} \eta_{\mu \nu} T\right) .
\end{aligned}
$$

Making use of the invariance under infinitesimal coordinate transformations, $x^{\mu} \rightarrow x^{\mu}+\xi^{\mu}$, where $\left|\partial_{\mu} \xi^{\nu}\right|$ is of the same order as $\left|h_{\mu \nu}\right|$, we choose the generalized 
Teyssandier gauge condition $Z_{\mu}=0[57,58]$. Hence, (9) becomes

$$
\begin{aligned}
& \left(\epsilon+\frac{1}{4} F_{2}(\square) \square\right)\left(\frac{1}{2} \square h_{\mu \nu}-\frac{1}{2(D-1)} \eta_{\mu \nu} R^{(1)}\right) \\
& =-8 \pi G\left(T_{\mu \nu}-\frac{1}{D-1} \eta_{\mu \nu} T\right) .
\end{aligned}
$$

Opposed to GR, the metric perturbation contains more d.o.f. than just a massless spin-2 field. Therefore, it turns out to be convenient to write the metric perturbation as

$$
h_{\mu \nu}=\epsilon\left(\eta_{\mu \nu} \phi+H_{\mu \nu}+\Psi_{\mu \nu}\right) \text {, }
$$

where $\phi$ resembles a massive spin- 0 field, $H_{\mu \nu}$ is a massless spin-2 field and $\Psi_{\mu \nu}$ is a massive spin-2 field. The distinction between $H_{\mu \nu}$ and $\Psi_{\mu \nu}$ will become clear in the following.

Inserting (11) into (10) and following the steps in [57, 58 ] one finds

$$
\left[\square-\epsilon m_{\phi}^{2}(\square)\right] \phi=\frac{16 \pi G}{(D-1)(D-2)} T,
$$

where the scalar field is defined as

$$
\phi \equiv \frac{1}{(D-1) m_{\phi}^{2}(\square)} R^{(1)},
$$

and

$$
m_{\phi}^{2}(\square) \equiv \frac{4(D-2)}{4(D-1) F_{1}(\square)+D F_{2}(\square)} .
$$

The field equations and the gauge condition for the massless spin-2 field are

$$
\begin{aligned}
\square \bar{H}_{\mu \nu} & =-16 \pi G T_{\mu \nu}, \\
\partial^{\mu} \bar{H}_{\mu \nu} & =0,
\end{aligned}
$$

where $\bar{H}_{\mu \nu}=H_{\mu \nu}-1 / 2 \eta_{\mu \nu} H$ and $H=\eta^{\mu \nu} H_{\mu \nu}$. Note that (13a) and (13b) lead to

$$
\partial^{\mu} T_{\mu \nu}=0
$$

to lowest order in $h_{\mu \nu}$.

For the massive spin-2 field one finds

$$
\begin{aligned}
{\left[\square-\epsilon m_{\Psi}^{2}(\square)\right] \Psi_{\mu \nu} } & =16 \pi G\left(T_{\mu \nu}-\frac{1}{D-1} \eta_{\mu \nu} T\right) \\
\partial_{\mu} \partial_{\nu} \Psi^{\mu \nu} & =\square \Psi
\end{aligned}
$$

where the massive spin-2 field is defined as

$$
\Psi_{\mu \nu} \equiv \frac{1}{m_{\Psi}^{2}}\left(\square h_{\mu \nu}-m_{\phi}^{2} \eta_{\mu \nu} \phi\right)
$$

and

$$
m_{\Psi}^{2}(\square) \equiv-\frac{4}{F_{2}(\square)} .
$$

It is useful to rewrite (15a) and (15b) as

$$
\begin{aligned}
{\left[\square-\epsilon m_{\Psi}^{2}(\square)\right] \hat{\Psi}_{\mu \nu} } & =16 \pi G T_{\mu \nu}, \\
\partial_{\mu} \partial_{\nu} \hat{\Psi}^{\mu \nu} & =0,
\end{aligned}
$$

where $\hat{\Psi}_{\mu \nu} \equiv \Psi_{\mu \nu}-\eta_{\mu \nu} \Psi$ and $\Psi=\eta^{\mu \nu} \Psi_{\mu \nu}$.

The distinction between $H_{\mu \nu}$ and $\Psi_{\mu \nu}$ can be easily understood in the case of quadratic gravity, where $m_{\Psi}(\square)=m_{\Psi}$ is independent of the d'Alembert operator and thus represents an honest mass term. In this case (15a) reduces to a massive Klein-Gordon equation, which shows that $\Psi_{\mu \nu}$ represents a massive wave. In addition, (13b) constrains $\bar{H}_{\mu \nu}$ by four conditions, whereas (15b) leads to only one condition on $\Psi_{\mu \nu}$. Hence, as usual for a massive wave, $\Psi_{\mu \nu}$ carries three additional d.o.f. The case we consider here represents a generalization of quadratic gravity because $m_{\Psi}(\square)$ depends on the d'Alembert operator.

Besides that, we observe that for $m_{\phi} \rightarrow \infty$ and $m_{\Psi} \rightarrow$ $\infty$ the spin-0 field and massive spin-2 field become nondynamical. Hence, only the massless spin-2 field represents a dynamical d.o.f. For $D=4$ and $\epsilon=+1$ this represents the GR limit. Conformal gravity is reproduced by $D=4, \epsilon=-1, m_{\Psi}(\square)=m_{\Psi}$, and $m_{\phi} \rightarrow \infty$. In this case there is no propagating scalar field, but a massless and a massive spin-2 field.

\section{SOLUTIONS}

Using the method of Green's function, the solutions to (12a), (13a), and (16a) can be written as

$$
\begin{aligned}
\phi & =\frac{16 \pi G}{(D-1)(D-2)} \int \mathrm{d}^{D} x^{\prime} \mathcal{G}_{\phi}\left(x-x^{\prime}\right) T\left(x^{\prime}\right), \\
\bar{H}_{\mu \nu} & =-16 \pi G \int \mathrm{d}^{D} x^{\prime} \mathcal{G}_{H}\left(x-x^{\prime}\right) T_{\mu \nu}\left(x^{\prime}\right), \\
\hat{\Psi}_{\mu \nu} & =16 \pi G \int \mathrm{d}^{D} x^{\prime} \mathcal{G}_{\Psi}\left(x-x^{\prime}\right) T_{\mu \nu}\left(x^{\prime}\right) .
\end{aligned}
$$

The propagators $\mathcal{G}_{\phi}, \mathcal{G}_{H}$, and $\mathcal{G}_{\Psi}$ are defined by

$$
\begin{aligned}
{\left[\square-\epsilon m_{\phi}^{2}(\square)\right] \mathcal{G}_{\Phi}\left(x-x^{\prime}\right) } & =\delta^{(D)}\left(x-x^{\prime}\right), \\
\square \mathcal{G}_{H}\left(x-x^{\prime}\right) & =\delta^{(D)}\left(x-x^{\prime}\right), \\
{\left[\square-\epsilon m_{\Psi}^{2}(\square)\right] \mathcal{G}_{\Psi}\left(x-x^{\prime}\right) } & =\delta^{(D)}\left(x-x^{\prime}\right) .
\end{aligned}
$$

Contracting (17c) with a partial derivative yields

$$
\begin{aligned}
\partial^{\mu} \hat{\Psi}_{\mu \nu}= & \int_{V} d^{D} x^{\prime}\left(\frac{\partial}{\partial x_{\mu}} \mathcal{G}_{\Psi}\left(x-x^{\prime}\right)\right) T_{\mu \nu}\left(x^{\prime}\right) \\
= & -\int_{V} d^{D} x^{\prime}\left(\frac{\partial}{\partial x_{\mu}^{\prime}} \mathcal{G}_{\Psi}\left(x-x^{\prime}\right)\right) T_{\mu \nu}\left(x^{\prime}\right) \\
= & -\left.\mathcal{G}_{\Psi}\left(x-x^{\prime}\right) T_{\mu \nu}\left(x^{\prime}\right)\right|_{\partial V} \\
& +\int_{V} d^{D} x^{\prime} \mathcal{G}_{\Psi}\left(x-x^{\prime}\right)\left(\frac{\partial}{\partial x_{\mu}^{\prime}} T_{\mu \nu}\left(x^{\prime}\right)\right) \\
= & 0
\end{aligned}
$$


where we have used $\frac{\partial}{\partial x^{\mu}} \mathcal{G}_{\Psi}\left(x-x^{\prime}\right)=-\frac{\partial}{\partial x^{\prime \mu}} \mathcal{G}_{\Psi}\left(x-x^{\prime}\right)$ for the second equal sign and integration by parts for the third equal sign. Furthermore, we have chosen a Ddimensional integration volume $V$ that is larger than the source, such that $T_{\mu \nu}(x)$ vanishes on its boundary $\partial V$. The last expression vanishes due to energy-momentum conservation given in (14).

Using

$\int \frac{\mathrm{d}^{D-1} k}{(2 \pi)^{D-1}} g(|\mathbf{k}|) e^{i \mathbf{k} \cdot \mathbf{r}}=\frac{1}{(2 \pi)^{\frac{D-1}{2}} r^{\frac{D-3}{2}}} \int_{0}^{\infty} \mathrm{d} y y^{\frac{D-1}{2}} g(y) J_{\frac{D-3}{2}}(y r)$,

where $g(x)$ is an arbitrary function and $J_{n}$ are the Bessel functions of the first kind. The frequency domain propagators for $D \geq 4$, given (17a)-(17c), can be written as

$$
\begin{aligned}
\tilde{\mathcal{G}}_{\phi}\left(\omega, \mathbf{x}-\mathbf{x}^{\prime}\right)= & \frac{1}{(2 \pi)^{\frac{D-1}{2}}\left|\mathbf{x}-\mathbf{x}^{\prime}\right|^{\frac{D-3}{2}}} \int_{0}^{\infty} \mathrm{d} k \\
& \times \frac{k^{\frac{D-1}{2}}}{\omega^{2}-k^{2}-\epsilon m_{\phi}^{2}(\square)} J_{\frac{D-3}{2}}\left(k\left|\mathbf{x}-\mathbf{x}^{\prime}\right|\right),
\end{aligned}
$$

$$
\begin{aligned}
\tilde{\mathcal{G}}_{H}\left(\omega, \mathbf{x}-\mathbf{x}^{\prime}\right)= & \frac{1}{(2 \pi)^{\frac{D-1}{2}}\left|\mathbf{x}-\mathbf{x}^{\prime}\right|^{\frac{D-3}{2}}} \int_{0}^{\infty} \mathrm{d} k \\
& \times \frac{k^{\frac{D-1}{2}}}{\omega^{2}-k^{2}} J_{\frac{D-3}{2}}\left(k\left|\mathbf{x}-\mathbf{x}^{\prime}\right|\right), \quad(21 \mathrm{~b}) \\
\tilde{\mathcal{G}}_{\Psi}\left(\omega, \mathbf{x}-\mathbf{x}^{\prime}\right)= & \frac{1}{(2 \pi)^{\frac{D-1}{2}}\left|\mathbf{x}-\mathbf{x}^{\prime}\right|^{\frac{D-3}{2}}} \int_{0}^{\infty} \mathrm{d} k \\
& \times \frac{k^{\frac{D-1}{2}}}{\omega^{2}-k^{2}-\epsilon m_{\Psi}^{2}(\square)} J_{\frac{D-3}{2}}\left(k\left|\mathbf{x}-\mathbf{x}^{\prime}\right|\right) .
\end{aligned}
$$

Note that $m_{\phi}$ and $m_{\Psi}$ depend on the d'Alembert operator and hence on $\omega$ and $k$. Thus, we cannot calculate the $k$-integral in (21a) and (21c) without specifying $F_{1}$ and $F_{2}$ or, equivalently, $m_{\phi}$ and $m_{\Psi}$. To analyze the radiation behavior of these three fields, in the following we restrict to the case in which $F_{1}(\square)=F_{1}$ and $F_{2}(\square)=F_{2}$ are independent of the d'Alembert operator (and hence independent of $\omega$ and $k$ ). This represents the case of quadratic gravity. The massless spin-2 field is well known from GR and hence we only derive the solutions to the spin-0 and the massive spin-2 field equations.

\section{A. The Massive Spin-0 Field}

Inserting (21a) into (17a) for $D=4$ yields

$$
\begin{aligned}
\phi(t, \mathbf{x})= & \frac{8 \pi G}{3} \int \mathrm{d}^{3} x^{\prime} \int \frac{\mathrm{d} \omega}{2 \pi} \int_{0}^{\infty} \frac{\mathrm{d} k}{(2 \pi)^{3 / 2}} \frac{k^{3 / 2}}{\left(\omega^{2}-k^{2}-\epsilon m_{\phi}^{2}\right)} \\
& \times \frac{J_{1 / 2}\left(k\left|\mathbf{x}-\mathbf{x}^{\prime}\right|\right)}{\left|\mathbf{x}-\mathbf{x}^{\prime}\right|^{1 / 2}} \tilde{T}\left(\omega, \mathbf{x}^{\prime}\right)
\end{aligned}
$$

where $m_{\phi}^{2}=2 /\left(3 F_{1}+F_{2}\right)$ and $J_{1 / 2}\left(k\left|\mathbf{x}-\mathbf{x}^{\prime}\right|\right)=$ $\left(2 / \pi k\left|\mathbf{x}-\mathbf{x}^{\prime}\right|\right)^{1 / 2} \sin \left(k\left|\mathbf{x}-\mathbf{x}^{\prime}\right|\right)$.

For $\epsilon=+1$ and $m_{\phi}^{2}>\omega^{2}$ (large mass of the massive spin-0 mode) the poles of (22) are on the imaginary $k$ axis. This leads to an exponential suppression for the massive spin-0 mode and to a negligible contribution to the gravitational radiation (see [56] for details). Hence, we do not study this case here. $\epsilon=-1$ and $m_{\phi}^{2}>\omega^{2}$ leads to an oscillating gravitational potential, which we also do not want to study here.

For $m_{\phi}^{2}<\omega^{2}$ (small mass of the massive spin-0 field) the poles are on the real $k$-axis. This leads to a massive propagating mode. Calculating the $k$-integral and using the quadrupole expansion $\left(k_{\omega, \phi} \mathbf{x}^{\prime} \cdot \mathbf{n} \ll 1\right.$, where $k_{\omega, \phi}=$ $\sqrt{\omega^{2}-\epsilon m_{\phi}^{2}}$ and $\mathbf{n}=\mathbf{x} / r$, where $r=|\mathbf{x}|$, is the unit vector in $\mathbf{x}$-direction) in (22), the solution in the far-field (for $r \gg R$ we have $\left|\mathbf{x}-\mathbf{x}^{\prime}\right| \approx r-\mathbf{x}^{\prime} \cdot \mathbf{n}+O\left(R^{2} / r\right)$, where $R$ is the typical spatial scale of the source) is given by

$$
\begin{aligned}
\phi(t, \mathbf{x}) & =-\frac{8 \pi G}{3} \int \mathrm{d}^{3} x^{\prime} \int \frac{\mathrm{d} \omega}{2 \pi} \frac{e^{i k_{\omega, \phi}\left|\mathbf{x}-\mathbf{x}^{\prime}\right|} \theta\left(\omega-m_{\phi}\right)+e^{-i k_{\omega, \phi}\left|\mathbf{x}-\mathbf{x}^{\prime}\right|} \theta\left(-\omega-m_{\phi}\right)}{4 \pi\left|\mathbf{x}-\mathbf{x}^{\prime}\right|} \tilde{T}\left(\omega, \mathbf{x}^{\prime}\right) e^{-i \omega t} \\
& =-\frac{G}{3 \pi r} \int \mathrm{d}^{3} x^{\prime}\left[\int_{m_{\phi}}^{\infty} \mathrm{d} \omega e^{-i \omega t} e^{i k_{\omega, \phi} r}\left(1-i k_{\omega, \phi} \mathbf{x}^{\prime} \cdot \mathbf{n}-\frac{k_{\omega, \phi}^{2}}{2}\left(\mathbf{x}^{\prime} \cdot \mathbf{n}\right)^{2}\right) \tilde{T}\left(\omega, \mathbf{x}^{\prime}\right)+\int_{-\infty}^{-m_{\phi}} \cdots\right]
\end{aligned}
$$

where the $\int_{-\infty}^{-m_{\phi}}$-contribution will be suppressed in the steps below, because its analysis is the same as for the first integral. The second line is exact up to the quadrupole and the far-field approximation.
It is convenient to define the mass-energy moments

$$
\begin{aligned}
M(t) & =\int \mathrm{d}^{3} x T^{00}(t, \mathbf{x}), \\
D^{i}(t) & =\int \mathrm{d}^{3} x x^{i} T^{00}(t, \mathbf{x}), \\
M^{i j}(t) & =\int \mathrm{d}^{3} x x^{i} x^{j} T^{00}(t, \mathbf{x}) .
\end{aligned}
$$


These quantities are called monopole, dipole, and quadrupole moments and in frequency space we denote them by $\tilde{M}(\omega), \tilde{D}^{i}(\omega)$, and $\tilde{M}^{i j}(\omega)$. Using these in (23) together with the relations (A10)-(A12) we get

$$
\begin{aligned}
\phi(t, \mathbf{x})= & -\frac{G}{3 \pi r} \int_{m_{\phi}}^{\infty} \mathrm{d} \omega e^{-i \omega t} e^{i k_{\omega, \phi} r}\left(-\tilde{M}(\omega)+i k_{\omega, \phi} n_{k} \tilde{D}^{k}(\omega)\right. \\
& \left.+\frac{k_{\omega, \phi}^{2}}{2} n_{k} n_{l} \tilde{M}^{k l}(\omega)-\frac{\omega^{2}}{2} \tilde{M}_{i}^{i}(\omega)\right) .
\end{aligned}
$$

For simplicity, we make the assumptions that $m_{\phi}^{2} / \omega^{2} \ll$ 1. Taking the time derivative and using $k_{\omega, \phi} \approx|\omega|(1-$ $\left.\epsilon \frac{m_{\phi}^{2}}{2 \omega^{2}}\right)$ for $m_{\phi}^{2} / \omega^{2} \ll 1$ leads to

$$
\begin{aligned}
\dot{\phi}(t, \mathbf{x}) \approx & -\frac{G}{3 \pi r} \int_{m_{\phi}}^{\infty} \mathrm{d} \omega e^{-i \omega t} e^{i k_{\omega, \phi} r}\left(i \omega \tilde{M}(\omega)+\omega^{2} n_{k} \tilde{D}^{k}(\omega)\right. \\
& \left.-i \frac{\omega^{3}}{2} n_{k} n_{l} \tilde{M}^{k l}(\omega)+i \frac{\omega^{3}}{2} \tilde{M}_{i}^{i}(\omega)\right)
\end{aligned}
$$

where the dot denotes the time derivative.

For a binary system with masses $m_{1}$ and $m_{2}$ on a circular orbit in the Newtonian limit, the contribution from the quadrupole moment can be described in the center of mass frame as originating from one particle with the reduced mass $\mu=m_{1} m_{2} /\left(m_{1}+m_{2}\right)$. Assuming the orbit to be in the xy-plane, the nonvanishing components of the quadrupole moment in the frequency domain are given by

$$
\begin{aligned}
& \tilde{M}_{11}(\omega)=\frac{\mu R^{2} \pi}{2}\left[\delta(\omega)-\delta\left(\omega+2 \omega_{s}\right)-\delta\left(\omega-2 \omega_{s}\right)\right] \\
& \tilde{M}_{22}(\omega)=\frac{\mu R^{2} \pi}{2}\left[\delta(\omega)+\delta\left(\omega+2 \omega_{s}\right)+\delta\left(\omega-2 \omega_{s}\right)\right] \\
& \tilde{M}_{12}(\omega)=\frac{\mu R^{2} \pi}{2 i}\left[\delta\left(\omega-2 \omega_{s}\right)-\delta\left(\omega+2 \omega_{s}\right)\right] \\
& \tilde{M}_{i}^{i}(\omega)=\mu R^{2} \pi \delta(\omega)
\end{aligned}
$$

where $M_{i}^{i}=\delta^{i j} M_{i j}$ is the spatial trace of the quadrupole moment and $\omega_{s}>0$ is the orbital frequency. The dipole moment can be written as

$$
D^{k}=m x_{c m}^{k}
$$

where $m=m_{1}+m_{2}$ is the total mass and $x_{c m}^{k}=\left(m_{1} x_{1}^{k}+\right.$ $\left.m_{2} x_{2}^{k}\right) / m$ is the $k$-th component of the center of mass coordinate. Hence, in the center of mass frame the dipole moment vanishes

$$
\tilde{D}^{k}(\omega)=0
$$

The monopole moment is given by

$$
\tilde{M}(\omega)=m \delta(\omega)
$$

Using (27a)-(27d), (29) and (30) in (26), we see that the monopole moment, the dipole moment and the trace of the quadrupole moment vanish and only the quadrupole contribution survives

$$
\dot{\phi}(t, \mathbf{x}) \approx \frac{i G}{3 \pi r} \int_{m_{\phi}}^{\infty} \mathrm{d} \omega e^{-i \omega t} e^{i k_{\omega, \phi} r} \frac{\omega^{3}}{2} n_{k} n_{l} \tilde{M}^{k l}(\omega) .
$$

The radiated energy in a three-dimensional volume $V$ larger than the source can be calculated by

$$
\dot{E}=r^{2} \int_{\partial V} \mathrm{~d} \Omega n_{s} T_{G R A V}^{s 0},
$$

where $\dot{E}$ is the time derivative of the gravitational energy, $T_{G R A V}^{\mu \nu}$ is the gravitational energy-momentum tensor and $d \Omega=\sin \theta d \theta d \phi$ is the differential solid angle. In (31) we have shown that monopole and dipole moments do not contribute to time derivatives of the massive spin0 field, but $T_{G R A V}^{s 0}$ can also contain terms with spatial derivatives like $\partial^{0} \partial^{s} h_{\rho \sigma} \square h^{\rho \sigma}$. By inserting (27a)-(27c) into (31) we can derive the relation

$$
\partial^{s} \phi=\partial^{0} \phi\left[1-\epsilon m_{\phi}^{2} /\left(8 \omega_{s}^{2}\right)+\mathcal{O}\left(m_{\phi}^{4} / \omega_{s}^{4}\right)\right] n^{s}+\mathcal{O}\left(1 / r^{2}\right) .
$$

This shows that spatial derivatives can be rewritten to time derivatives to leading order in $m_{\phi}^{2} / \omega_{s}^{2}$ and that monopole and dipole radiation do not contribute to (32).

\section{B. The Massive Spin-2 Field}

In (19) we have shown that the harmonic gauge condition arises dynamically for the massive spin-2 field and hence in the presence of a source only the transverse modes of the massive spin- 2 field are excited. Thus, we can use the residual gauge freedom to bring the massive spin-2 field to the transverse traceless (TT) gauge $\partial^{\nu} \Psi_{\mu \nu}^{T T}=0, \Psi_{0 \mu}^{T T}=0$ and, $\Psi^{T T}=0$, meaning that the massless and massive spin-2 fields are constrained by the same number of conditions. This fundamentally affects the gravitational radiation behavior in this model of higher derivative gravity. In vacuum quadratic gravity contains eight d.o.f.: one from the massive scalar field, two from the massless spin-2 field, and five from the massive spin-2 field. But GWs created by a source carry only five propagating d.o.f. since three of the five massive modes are not excited. Additionally, monopole and dipole radiation vanish. To see this, we look at $(17 \mathrm{c})$ for $D=4$ and $m_{\Psi}(\square)=m_{\Psi}$, which is given by

$$
\begin{aligned}
\hat{\Psi}_{\mu \nu}(t, \mathbf{x})= & 16 \pi G \int \mathrm{d}^{3} x^{\prime} \int \frac{\mathrm{d} \omega}{2 \pi} \int_{0}^{\infty} \frac{\mathrm{d} k}{(2 \pi)^{3 / 2}} \\
& \times \frac{k^{3 / 2}}{\left(\omega^{2}-k^{2}-\epsilon m_{\Psi}^{2}\right)} \frac{J_{1 / 2}\left(k\left|\mathbf{x}-\mathbf{x}^{\prime}\right|\right)}{\left|\mathbf{x}-\mathbf{x}^{\prime}\right|^{(1 / 2)}} \\
& \times \tilde{T}_{\mu \nu}\left(\omega, \mathbf{x}^{\prime}\right),
\end{aligned}
$$

where $m_{\Psi}^{2}=-4 / F_{2}$ is independent of $\omega$ and $k$.

For the same reasons as for the spin-0 field we only study the case $m_{\Psi}^{2}<\omega^{2}$ (small mass of the massive spin2 field), which leads to a propagating wave and no oscillations in the gravitational potential. 
Calculating the $k$-integral and using the quadrupole expansion $\left(k_{\omega, \Psi} \mathbf{x}^{\prime} \cdot \mathbf{n} \ll 1\right.$, where $\left.k_{\omega, \Psi}=\sqrt{\omega^{2}-\epsilon m_{\Psi}^{2}}\right)$ in (34), the solution in the far field is given by

$$
\begin{aligned}
\hat{\Psi}_{\mu \nu}(t, \mathbf{x})= & -\frac{4 G}{r} \int \mathrm{d}^{3} x^{\prime}\left[\int_{m_{\Psi}}^{\infty} \frac{\mathrm{d} \omega}{2 \pi} e^{-i \omega t} e^{i k_{\omega, \Psi} r}\right. \\
& \times\left(1-i k_{\omega, \Psi} \mathbf{x}^{\prime} \cdot \mathbf{n}-\frac{k_{\omega, \Psi}^{2}}{2}\left(\mathbf{x}^{\prime} \cdot \mathbf{n}\right)^{2}\right) \\
& \left.\times \tilde{T}_{\mu \nu}\left(\omega, \mathbf{x}^{\prime}\right)+\int_{-\infty}^{-m_{\Psi}} \cdots\right] .
\end{aligned}
$$

The second integral in the square brackets is suppressed in the steps below, because its analysis is analogous to the first integral. This expression is exact in the quadrupole approximation and the far-field approximation. Using (A10)-(A12) we can write the components of (35) as

$$
\begin{aligned}
\hat{\Psi}^{00}= & -\frac{4 G}{r} \int_{m_{\Psi}}^{\infty} \frac{\mathrm{d} \omega}{2 \pi} e^{-i \omega t} e^{i k_{\omega, \Psi} r}\left(\tilde{M}(\omega)-i k_{\omega, \Psi} n_{k} \tilde{D}^{k}(\omega)\right. \\
& \left.-\frac{k_{\omega, \Psi}^{2}}{2} n_{k} n_{l} \tilde{M}^{k l}(\omega)\right) \\
\hat{\Psi}^{0 i}= & -\frac{4 G}{r} \int_{m_{\Psi}}^{\infty} \frac{\mathrm{d} \omega}{2 \pi} e^{-i \omega t} e^{i k_{\omega, \Psi} r} \\
& \times\left(-i \omega \tilde{D}^{i}(\omega) \times-\frac{\omega}{2} k_{\omega, \Psi} n_{k} \tilde{M}^{k i}(\omega)\right) \\
\hat{\Psi}^{i j}= & \frac{2 G}{r} \int_{m_{\Psi}}^{\infty} \frac{\mathrm{d} \omega}{2 \pi} e^{-i \omega t} e^{i k_{\omega, \Psi} r} \omega^{2} \tilde{M}^{i j}(\omega) .
\end{aligned}
$$

We could use (29) to set the dipole contribution to zero, but it is instructive to keep it and to show that it also vanishes due to the dynamically induced gauge condition (19). Note that we can expand $k_{\omega, \Psi} \approx|\omega|\left(1-\epsilon \frac{m_{\Psi}^{2}}{2 \omega^{2}}\right)$ for $m_{\Psi}^{2} / \omega^{2} \ll 1$. Using this expansion, the time derivatives of (36a)-(36c) simplify to

$$
\begin{aligned}
\dot{\hat{\Psi}}^{00} \approx & -\frac{4 G}{r} \int_{m_{\Psi}}^{\infty} \frac{\mathrm{d} \omega}{2 \pi} e^{-i \omega t} e^{i k_{\omega, \Psi} r}\left(-i \omega \tilde{M}(\omega)-\omega^{2} n_{k} \tilde{D}^{k}(\omega)\right. \\
+ & \left.i \frac{\omega^{3}}{2} n_{k} n_{l} \tilde{M}^{k l}(\omega)\right), \\
\dot{\hat{\Psi}}^{0 i} \approx & -\frac{4 G}{r} \int_{m_{\Psi}}^{\infty} \frac{\mathrm{d} \omega}{2 \pi} e^{-i \omega t} e^{i k_{\omega, \Psi} r} \\
& \times\left(-\omega^{2} \tilde{D}^{i}(\omega)+i \frac{\omega^{3}}{2} n_{k} \tilde{M}^{k i}(\omega)\right), \\
\dot{\hat{\Psi}}^{i j} & \approx-i \frac{2 G}{r} \int_{m_{\Psi}}^{\infty} \frac{\mathrm{d} \omega}{2 \pi} e^{-i \omega t} e^{i k_{\omega, \Psi} r} \omega^{3} \tilde{M}^{i j}(\omega) .
\end{aligned}
$$

Inserting (36a)-(36c) explicitly into (19) leads to

$$
\begin{aligned}
& -i \int_{m_{\Psi}}^{\infty} \frac{\mathrm{d} \omega}{2 \pi} e^{-i \omega t} e^{i k_{\omega, \Psi} r} \omega \tilde{M}(\omega)=0, \\
& -\int_{m_{\Psi}}^{\infty} \frac{\mathrm{d} \omega}{2 \pi} e^{-i \omega t} e^{i k_{\omega, \Psi} r} \omega^{2} \tilde{D}^{i}(\omega)=0 .
\end{aligned}
$$

Using (38a) and (38b) in (37a)-(37c), we see that monopole and dipole contributions vanish and only the quadrupole moment contributes. Note that this is a consequence of the conservation of the energy-momentum tensor in the linearized theory; see (14). In contrast to GR, this result is approximate because in (37a)-(37c) we used $k_{\omega, \Psi} \approx|\omega|$.

Since the massive spin-2 field can be brought to the TT gauge and invoking (27a)-(27c), the relevant components for the gravitational radiation are (note that we have to consider the $\int_{-\infty}^{-m_{\Psi}} \ldots$-contribution here)

$$
\begin{array}{r}
\hat{\Psi}_{11}(t, r)=-\hat{\Psi}_{22}(t, r)=-\frac{4 G \mu R^{2} \omega_{s}^{2}}{r} \cos \left(2 \omega_{s} t_{m}\right), \\
\hat{\Psi}_{12}(t, r)=\hat{\Psi}_{21}(t, r)=-\frac{4 G \mu R^{2} \omega_{s}^{2}}{r} \sin \left(2 \omega_{s} t_{m}\right), \\
\hat{\Psi}_{i}^{i}(t, r)=\hat{\Psi}_{3 i}(t, r)=\hat{\Psi}_{i 3}(t, r)=0
\end{array}
$$

where $t_{m}=t-v_{m} r$ is the travel time and $v_{m}=$ $\sqrt{1-\epsilon m_{\Psi}^{2} /\left(4 \omega_{s}^{2}\right)}$ is the group velocity of the massive spin-2 field. From this it becomes clear that spatial derivatives can be related to time derivatives by

$\partial^{s} \hat{\Psi}^{\rho \sigma}=\partial^{0} \hat{\Psi}^{\rho \sigma}\left[1-\epsilon m_{\Psi}^{2} /\left(8 \omega_{s}^{2}\right)+\mathcal{O}\left(m_{\Psi}^{4} / \omega_{s}^{4}\right)\right] n^{s}+\mathcal{O}\left(1 / r^{2}\right)$.

Hence, all spatial derivatives, which appear in the radiated energy, can be replaced by time derivatives to lowest order in $m_{\Psi}^{2} / \omega_{s}^{2}$. This demonstrates that no energy is carried away in monopole and dipole radiation by the massive spin-2 mode.

\section{CONCLUSION}

In this work we discussed the degrees of freedom and the gravitational radiation in generalized higherderivative gravity and, in particular, in quadratic gravity. We derived the linearized field equations for the metric perturbation for $D$ dimensions and introduced the generalized Teyssandier gauge, which is convenient for higherderivative theories. It turned out to be useful to separate the metric perturbation in a massive spin-0 field, a massless spin-2 field, and a massive spin-2 field, which obey massless and massive wave equations. We have shown that the massive spin-2 field satisfies the harmonic gauge condition, which originates from the conservation of the matter energy-momentum tensor in linearized theory. In Sec. III we derived the solutions for the massive spin-0 and massive spin-2 field (the massless spin-2 field is well known from GR) by the methods of Green's function for $D=4$ and constant masses, which represents the case of quadratic gravity. In this case the metric perturbation carries eight degrees of freedom in general. After that, to study the energy which is carried by these modes, we applied the solutions to a binary system in circular motion and in the Newtonian limit. For the massive spin0 field and the massive spin- 2 field it turned out that 
there is no monopole and dipole radiation, but only the quadrupole moment contributes. For the massive spin-2 field monopole and dipole radiation vanish as a consequence of the dynamically induced harmonic gauge condition. This means that, as for the massless spin-2 field, for the massive spin-2 field only the two transverse and traceless modes are excited by a matter source.

\section{ACKNOWLEDGMENTS}

The author wishes to thank Dominik J. Schwarz for valuable discussions and suggestions on improving the manuscript, as well as Breno L. Giacchini and Gustatavo P. de Brito for useful hints on the literature. We acknowledge financial support from Deutsche Forschungsgemeinschaft (DFG) under Grant No. RTG 1620 "Models of Gravity". We also thank the COST Action CA15117 "Cosmology and Astrophysics Network for Theoretical Advances and Training Actions (CANTATA)", supported by COST (European Cooperation in Science and Technology).
[1] C. M. Will, Living Rev. Relativity 17, 4 (2014).

[2] A. Del Popolo and M. Le Delliou, Galaxies 5, 17 (2017), arXiv:1606.07790 [astro-ph.CO].

[3] P. Bull, Y. Akrami, J. Adamek, T. Baker, E. Bellini, J. B. Jiménez, E. Bentivegna, S. Camera, S. Clesse, J. H. Davis, et al., Phys. Dark Universe 12, 56 (2016).

[4] S. Weinberg, Rev. Mod. Phys. 61, 1 (1989).

[5] S. M. Carroll, Living Rev. Relativity 4, 1 (2001), arXiv:0004075 [astro-ph].

[6] R. Bousso, Gen. Relativ. Gravit. 40, 607 (2008), arXiv:0708.4231 [hep-th].

[7] M. Veltman et al., Ann. Inst. Henri Poincaré 20, 69 (1974).

[8] S. Deser and P. van Nieuwenhuizen, Phys. Rev. D 10, 401 (1974).

[9] K. Stelle, Gen. Relativ. Gravit. 9, 353 (1978).

[10] K. Stelle, Phys. Rev. D 16, 953 (1977).

[11] M. Asorey, J. Lopez, and I. Shapiro, Int. J. Mod. Phys. A 12, 5711 (1997).

[12] L. Modesto, Phys. Rev. D 86, 044005 (2012).

[13] L. Modesto, Astron. Rev. 8, 4 (2013).

[14] M. Ostrogradsky, Mem. Acad. St. Petersbourg 6, 385 (1850).

[15] R. P. Woodard, Scholarpedia 10, 32243 (2015), arXiv:1506.02210 [hep-th].

[16] P. D. Mannheim and A. Davidson, arXiv:0001115 [hepth].

[17] C. M. Bender, Rep. Prog. Phys. 70, 947 (2007).

[18] C. M. Bender and P. D. Mannheim, Phys. Rev. D 78, 025022 (2008).

[19] C. M. Bender and P. D. Mannheim, J. Phys. A 41, 304018 (2008).

[20] C. M. Bender and P. D. Mannheim, Phys. Rev. Lett. 100, 110402 (2008).

[21] P. D. Mannheim, Phil. Trans. R. Soc. A 371, 20120060 (2013).

[22] P. D. Mannheim, J. Phys. A 51, 315302 (2018), arXiv:1512.04915 [hep-th].

[23] P. D. Mannheim, Phys. Lett. B 753, 288 (2016).

[24] A. Salvio and A. Strumia, Eur. Phys. J. C 76, 227 (2016).

[25] A. Accioly, B. L. Giacchini, and I. L. Shapiro, Phys. Rev. D 96, 104004 (2017).

[26] E. Tomboulis, arXiv:9702146 [hep-th] .

[27] T. Biswas, A. Mazumdar, and W. Siegel, J. Cosmol. Astropart. Phys. 03 (2006) 009.
[28] T. Biswas, E. Gerwick, T. Koivisto, and A. Mazumdar, Phys. Rev. Lett. 108, 031101 (2012).

[29] L. Modesto and L. Rachwał, Nucl. Phys. B 889, 228 (2014).

[30] M. Raidal and H. Veermäe, Nuc. Phys. B 916, 607 (2017), arXiv:1611.03498 [hep-th].

[31] B. Zwiebach, Phys. Lett. B 156, 315 (1985).

[32] S. Deser and A. Redlich, Phys. Lett. B 176, 350 (1986).

[33] D. Jones and A. Lawrence, Z. Phys. C 42, 153 (1989).

[34] B. Abbott, R. Abbott, T. Abbott, M. Abernathy, F. Acernese, K. Ackley, C. Adams, T. Adams, P. Addesso, R. Adhikari, et al., Phys. Rev. Lett. 116, 061102 (2016).

[35] B. Abbott, R. Abbott, T. Abbott, M. Abernathy, F. Acernese, K. Ackley, C. Adams, T. Adams, P. Addesso, R. Adhikari, et al., Phys. Rev. Lett. 116, 241103 (2016).

[36] B. Abbott, R. Abbott, T. Abbott, F. Acernese, K. Ackley, C. Adams, T. Adams, P. Addesso, R. Adhikari, et al. (Ligo Scientific and Virgo Collaborations) Phys. Rev. Lett. 118, 221101 (2017).

[37] B. Abbott, R. Abbott, T. Abbott, F. Acernese, K. Ackley, C. Adams, T. Adams, P. Addesso, R. Adhikari, V. Adya, et al., Astrophys. J. Lett. 851, L35 (2017), arXiv:1711.05578 [astro-ph.HE].

[38] B. P. Abbott, R. Abbott, T. Abbott, F. Acernese, K. Ackley, C. Adams, T. Adams, P. Addesso, R. Adhikari, V. Adya, et al., Phys. Rev. Lett. 119, 141101 (2017).

[39] B. P. Abbott, R. Abbott, T. Abbott, F. Acernese, K. Ackley, C. Adams, T. Adams, P. Addesso, R. Adhikari, V. Adya, et al., Phys. Rev. Lett. 119, 161101 (2017).

[40] J. M. Weisberg and J. H. Taylor, Binary Radio Pulsars: Proc. Aspen Conference, ASP Conf. Series Vol. 328, edited by F. A. Rasio and I. H. Stairs (ASP, San Francisco, 2005), p. 25, arXiv:0407149 [astro-ph].

[41] B. Abbott, R. Abbott, R. Adhikari, A. Ananyeva, S. Anderson, S. Appert, K. Arai, M. Araya, J. Barayoga, B. Barish, et al., Astrophys. J. Lett. 848, L12 (2017).

[42] D. A. Coulter, R. J. Foley, C. D. Kilpatrick, M. R. Drout, A. L. Piro, B. J. Shappee, M. R. Siebert, J. D. Simon, N. Ulloa, D. Kasen, et al., Science 3581556 (2017).

[43] B. P. Abbott, R. Abbott, T. D. Abbott, F. Acernese, K. Ackley, C. Adams, T. Adams, P. Addesso, R. X. Adhikari, V. V. B. Adya, et al., Astrophys. J. Lett. 848, L13 (2017). 
[44] L. Lombriser and N. A. Lima, Phys. Lett. B 765, 382 (2017).

[45] T. Baker, E. Bellini, P. G. Ferreira, M. Lagos, J. Noller, and I. Sawicki, Phys. Rev. Lett. 119, 251301 (2017).

[46] P. Creminelli and F. Vernizzi, Phys. Rev. Lett. 119, 251302 (2017), arXiv:1710.05877 [astro-ph.CO].

[47] J. M. Ezquiaga and M. Zumalacárregui, Phys. Rev. Lett. 119, 251304 (2017), arXiv:1710.05901 [astro-ph.CO].

[48] J. Sakstein and B. Jain, Phys. Rev. Lett. 119, 251303 (2017).

[49] H. Nersisyan, N. A. Lima, and L. Amendola, arXiv:1801.06683 [astro-ph.CO].

[50] Y. Akrami, P. Brax, A.-C. Davis, and V. Vardanyan, Phys. Rev. D 97, 124010 (2018), arXiv:1803.09726 [astroph.CO].

[51] A. Accioly, B. L. Giacchini, and I. L. Shapiro, Eur. Phys. J. C 77, 540 (2017).

[52] B. L. Giacchini, Phys. Lett. B 766, 306 (2017).

[53] B. L. Giacchini and T. d. P. Netto, arXiv:1806.05664 [grqc].

[54] S. Deser and B. Tekin, Phys. Rev. D 67, 084009 (2003).

[55] W. Nelson, Phys. Rev. D 82, 104026 (2010).

[56] C. Caprini, P. Hölscher, and D. J. Schwarz, Phys. Rev. D 98, 084002 (2018), arXiv:1804.01876 [gr-qc].

[57] A. Accioly, J. de Almeida, G. P. de Brito, and W. Herdy, arXiv:1707.02083v1 [hep-th].

[58] J. de Almeida, G. P. de Brito, and F. A. Silveira, Teyssandier-like gauge condition for a general class of higher-derivative gravity (to be published).

[59] T. Biswas, A. Conroy, A. S. Koshelev, and A. Mazumdar, Classical Quantum Gravity 31, 015022 (2013).

\section{Appendix A: Conventions}

The signature of the metric is

$$
g=\operatorname{diag}(-,+,+,+, \cdots,+)
$$

containing one negative and $D-1$ positive entries. The Christoffel symbols are defined by

$$
\Gamma_{\kappa \mu}^{\lambda}=\frac{1}{2} g^{\lambda \rho}\left(\partial_{\kappa} g_{\rho \mu}+\partial_{\mu} g_{\rho \kappa}-\partial_{\rho} g_{\kappa \mu}\right)
$$

and the Riemann tensor is given by

$$
R_{\mu \nu \kappa}^{\lambda}=-\left(\partial_{\nu} \Gamma_{\mu \kappa}^{\lambda}-\partial_{\kappa} \Gamma_{\mu \nu}^{\lambda}+\Gamma_{\nu \alpha}^{\lambda} \Gamma_{\mu \kappa}^{\alpha}-\Gamma_{\kappa \alpha}^{\lambda} \Gamma_{\mu \nu}^{\alpha}\right)
$$

Contracting the first and the third index, we find the Ricci tensor

$$
R_{\mu \nu}=R_{\mu \rho \nu}^{\rho}
$$

The Ricci scalar is defined as

$$
R=g^{\mu \nu} R_{\mu \nu}
$$

The Einstein equations in the convention used in this work read

$$
G_{\mu \nu} \equiv R_{\mu \nu}-\frac{1}{2} g_{\mu \nu} R=-8 \pi G T_{\mu \nu}+\Lambda g_{\mu \nu} .
$$

A list of the curvature tensors to first order in $h_{\mu \nu}$ is given by

$$
\begin{aligned}
R_{\nu \rho \sigma}^{\mu(1)}=\frac{1}{2}\left(-\partial_{\nu} \partial_{\rho} h_{\sigma}^{\mu}-\partial^{\mu} \partial_{\sigma} h_{\nu \rho}+\partial^{\mu} \partial_{\rho} h_{\nu \sigma}+\partial_{\nu} \partial_{\sigma} h_{\rho}^{\mu}\right) & (\mathrm{A} 7) \\
R_{\mu \nu}^{(1)} & =\frac{1}{2}\left(\square h_{\mu \nu}-\partial_{\rho} \partial_{\mu} h_{\nu}^{\rho}-\partial_{\nu} \partial_{\rho} h_{\mu}^{\rho}+\partial_{\mu} \partial_{\nu} h\right), \quad(\mathrm{A} 8) \\
R^{(1)} & =\square h-\partial_{\mu} \partial_{\nu} h^{\mu \nu} .
\end{aligned}
$$

Further, we present useful relations between the energy-momentum tensor and the mass-energy moments using energy-momentum conservation in flat spacetime

$$
\begin{aligned}
\int \mathrm{d}^{D} x \tilde{T}^{i j}(\omega, \mathbf{x}) & =-\frac{\omega^{2}}{2} \int \mathrm{d}^{D} x x^{i} x^{j} \tilde{T}^{00}(\omega, \mathbf{x}) \\
& =-\frac{\omega^{2}}{2} \tilde{M}^{i j}(\omega) \\
\int \mathrm{d}^{D} x \tilde{T}^{0 i}(\omega, \mathbf{x}) & =-i \omega \int \mathrm{d}^{D} x x^{i} \tilde{T}^{00}(\omega, \mathbf{x}) \\
& =-i \omega \tilde{D}^{i}(\omega) \\
\int \mathrm{d}^{D} x \tilde{T}^{i j}(\omega, \mathbf{x}) & =-i \omega \int \mathrm{d}^{D} x x^{i} \tilde{T}^{j 0}(\omega, \mathbf{x}) \\
& =-\frac{\omega^{2}}{2} \tilde{M}^{i j}(\omega) .
\end{aligned}
$$

